\title{
Nanomedicine in Thrombosis
}

\section{Antonio Peramo*}

Department of Oral and Maxillofacial Surgery, University of Michigan, Ann Arbor, Michigan 48109, USA

There is an increased number of research articles - in the Journal of Nanomedicine and Nanotechnology and elsewhere - describing possible applications of nanomedicine in cardiovascular diseases. These diseases have an increased incidence in developed countries due to an aging population and will also gradually increase in developing countries. In general, the nanomedical applications described in the literature have been focusing on imaging and therapy of atherosclerosis [1] and in vivo and ex-vivo identification and marking of vascular biosignatures [2], to name just two examples.

Thrombosis is, however, an area of cardiovascular research that would substantially benefit of novel and sustained nanotechnology approaches. This area is not being investigated and opportunities abound for nanotechnologists and biomedical researchers to collaborate to advance nanomedical solutions for improving diagnosis and understanding progression and recurrence of thrombosis [3] During thrombosis there is a formation of blood clots with the vessels that lead to arterial or vein obstruction, inflammation, as well as the production of pro-coagulant vesicles known as microparticles, released primarily from platelets.

Given that the field is so widely open we will mention a few interesting applications, but the list is obviously much larger. While the toxicological effects of synthetic nanoparticles that are starting to be used in nanomedicine, like quantum dots or carbon nanotubes, have been under study for some time, the involvement of these or other nanoparticles in thrombosis and their effects are mostly unknown. A few recent studies have shown that surface carboxylated quantum dots may cause vascular thrombosis in the pulmonary circulation [4] or that carbon nanotubes accelerate thrombus formation in the circulation [5] and activate platelets [6]. Additional studies should be necessary, particularly in patient populations at risk of thrombotic diseases.

In nanomedicine it is common to use the procedures to deliver cargo (drugs, DNA or other molecules) to selected cell types and tissues [7]. These techniques should also be explored and implemented in circulating and in the endothelial cells known to have an active participation in thrombosis. Methods for the intracellular delivery of markers to cells and vesicles involved in thrombosis may provide critical information for their identification and tracking, and will make possible to perform studies that requires leaving intact (unmodified) the outer membrane of the cells. Implementation of these methods will certainly be easier in animal model of thrombosis [8] where nanoparticles can be used in in vivo localization, tracking of cells in thrombus formation and selective targeting/identification of cells as well as in ex vivo identification of markers for prediction of thrombotic events.

Unlike regular drug delivery and molecular therapeutic models, nanomedicine allows the development of a variety of nanoagents that can perform multiple tasks in the early identification and resolution of disease. In essence, we expect this emerging field of nanomedicine in thrombosis to meet the demand for innovative approaches in the diagnosis and treatment of the complications associated with this disease.

\section{References}

1. Nahrendorf M, Zhang H, Hembrador S, Panizzi P, Sonsnovik DE, et al. (2008) Nanoparticle PET-CT imaging of macrophages in inflammatory atherosclerosis. Circulation 117: 379-387.

2. Pan D, Williams TA, Senpan A, Allen JS, Scott MJ, et al. (2009) Detecting vascular biosignatures with a colloidal, radio-opaque polymeric nanoparticle. $J$ Am Chem Soc 131: 15522-15527.

3. Peramo A (2012) Membranes 2: 1-15.

4. Geys J, Nemmar A, Verbeken E, Smolders E, Ratoi M, et al. (2008) Acute toxicity and prothrombotic effects of quantum dots: impact of surface charge. Environ Health Perspect 116: 1607-1613.

5. Bihari P, Holzer M, Praetner M, Fent J, Lerchenberger M, et al. (2010) Singlewalled carbon nanotubes activate platelets and accelerate thrombus formation in the microcirculation. Toxicology 269: 148-154.

6. Semberova J, De Paoli Lacerda SH, Simakova O, Holada K, Gelderman MP, et al. (2009) Carbon nanotubes activate blood platelets by inducing extracellular Ca2+ influx sensitive to calcium entry inhibitors. Nano Lett 9: 3312-3317.

7. Sahay G, Alakhova DY, Kabanov AV (2010) Endocytosis of nanomedicines. J Control Release 145: 182-195.

8. Diaz JA, Obi AT, Myers DD Jr, Wrobleski SK, Henke PK, et al. (2012) Critica review of mouse models of venous thrombosis. Arterioscler Thromb Vasc Biol 32: $556-562$
*Corresponding author: Antonio Peramo, Department of Oral and Maxillofacial Surgery, University of Michigan, Ann Arbor, Michigan 48109, USA, E-mail: aperamo@umich.edu

Received April 06, 2012; Accepted April 12, 2012; Published April 16, 2012

Citation: Peramo A (2012) Nanomedicine in Thrombosis. J Nanomedic Nanotechnol 3:e106. doi:10.4172/2157-7439.1000e106

Copyright: (c) 2012 Peramo A. This is an open-access article distributed under the terms of the Creative Commons Attribution License, which permits unrestricted use, distribution, and reproduction in any medium, provided the original author and source are credited. 